\title{
A Plea for Smoking-Free Policies in COVID-19 Times: Cardiovascular Prevention as an Ally in Coronavirus Containment
}

\author{
Giacomo Tini $^{1}$ (I) $\cdot$ Giovanna Gallo $^{1} \cdot$ Beatrice Musumeci ${ }^{1} \cdot$ Giuliano Tocci $^{1} \cdot$ Massimo Volpe $^{1}$
}

Received: 27 February 2021 / Accepted: 22 March 2021 / Published online: 26 April 2021

(c) Italian Society of Hypertension 2021

The novel coronavirus disease 2019 (COVID-19) pandemic has required the whole world to adopt stringent measures of social distancing and to change common habits in order to prevent the spreading of the infection across the population. The use of universal facial masking is the most emblematic intervention currently undertaken by several countriesand represent a simple, yet extremely effective measure to protect the self and others from COVID-19 spreading [1]. However, in everyday life the use of facial masking may be limited. A common example is cigarette smoking. Regulations indicating to wear facial mask are easily violated in public areas, where, even though respecting social distancing, people can still smoke.

To date, several reports have highlighted how smoking supports COVID-19 transmission and is associated with a more severe COVID-19 syndrome [2]. Accordingly, the importance of promoting smoking cessation in the pandemic era has been acknowledged [3].

These data add to another well-known reality. Smoking remains the leading preventable cause of death-to put things in perspective, it account for half a million deaths in the United States every year [4], and significantly contribute to cardiovascular disease burden worldwide. Policies aimed at reducing prevalence of smokers remain a priority for the health community.

The issue of smoking has thus doubled its detrimental effects during this pandemic time-yet, there is still plenty of room for implementation of smoking-free legislations, which may prove a double benefit. In this very moment, reinforcing prohibition of smoking in public areas may help supporting facial masking compliance and reducing COVID-19 transmission. Moreover, it would represent an

Giacomo Tini

giacomotini@outlook.it

1 Cardiology, Clinical and Molecular Medicine Department, Sant'Andrea Hospital, Sapienza University of Rome, Via di Grottarossa, 1035-1038, 00189 Rome, Italy important investment for the future, to contrast the global burden of cardiovascular diseases [5]. Cardiovascular prevention is not a secondary issue, as it is related to COVID-19 in many ways. The toll of COVID-19 has been shown to be significantly higher among subjects affected by cardiovascular diseases; and, at the same time, periods of lockdown and reduced accessibility to healthcare services due to the sanitary emergency have had a deleterious impact on the awareness and management of cardiovascular risk factors in the community [3]. In particular, anxiety, frustration and difficulty to cope with social isolation due to lockdown periods may predispose to start (or re-start) smoking habits [3]. Hence, a focus on cardiovascular prevention appears strongly needed to tackle direct and indirect consequences of COVID-19, in the short and long-term.

Promoting smoking cessation and cardiovascular prevention should be considered a priority, even (and especially!) in current times, and be pursued by medical societies and by politics.

Acknowledgements This article has been endorsed by the Italian Society of Cardiovascular Prevention (SIPREC).

\section{Declarations}

Conflict of interest On behalf of all authors, the corresponding author states that there is no conflict of interest.

\section{References}

1. Brooks JT, Butler JC, Redfield RR. Universal masking to prevent SARS-CoV-2 transmission-the time is now. JAMA. 2020;324:635.

2. Berlin I, Thomas D, Le Faou A-L, Cornuz J. COVID-19 and smoking. Nicotine Tob Res. 2020;22:1650-2.

3. Volpe M, Battistoni A, Board of the Italian Society of Cardiovascular Prevention, Bellotti P, Bellone S, Bertolotti M, et al. Recommendations for cardiovascular prevention during the SARSCoV-2 pandemic: an executive document by the Board of the 
Italian Society of Cardiovascular Prevention. High Blood Press Cardiovasc Prev. 2020;27:373-7.

4. Proctor RN. The cigarette: a political history-the past, present, and future of US tobacco. JAMA. 2020;324:10
5. Joseph P, Leong D, McKee M, Anand SS, Schwalm J-D, Teo K, et al. Reducing the global burden of cardiovascular disease, part 1: the epidemiology and risk factors. Circ Res. 2017;121:677-94. 